\title{
Erratum to: Differences in the activity pattern of the wild boar Sus scrofa related to human disturbance
}

\author{
Haruka Ohashi • Masae Saito • Reiko Horie • Hiroshi Tsunoda $\cdot$ Hiromu Noba • \\ Haruka Ishii • Takashi Kuwabara • Yutaka Hiroshige • Shinsuke Koike • Yoshinobu Hoshino • \\ Hiroto Toda $\cdot$ Koichi Kaji
}

Published online: 13 April 2014

(C) Springer-Verlag Berlin Heidelberg 2014

Erratum to: Eur J Wildl Res (2013) 59:167-177

DOI 10.1007/s10344-012-0661-z

The original version of this article inadvertently contained a mistake. An incorrect version of Table 2 was published. The correct table appears below.
The online version of the original article can be found at http:// dx.doi:10.1007/s10344-012-0661-z.

H. Ohashi $(\bowtie) \cdot$ M. Saito $\cdot$ T. Kuwabara $\cdot$ Y. Hiroshige

Education and Research Center for Frontier Agricultural Science,

Tokyo University of Agriculture and Technology, 3-5-8 Saiwai-cho,

Fuchu, Tokyo 183-8509, Japan

e-mail: oharu0429@gmail.com

\section{R. Horie}

Faculty of Agriculture, Utsunomiya University, 350 Mine-machi,

Utsunomiya, Tochigi 321-8505, Japan

\section{H. Tsunoda}

Faculty of Agriculture, Tokyo University of Agriculture and

Technology, 3-5-8 Saiwai-cho, Fuchu, Tokyo 183-8509, Japan

H. Noba $\cdot$ H. Ishii

Graduate School of Agriculture, Tokyo University of Agriculture and

Technology, 3-5-8 Saiwai-cho, Fuchu, Tokyo 183-8509, Japan

\section{S. Koike $\cdot$ Y. Hoshino $\cdot$ H. Toda $\cdot$ K. Kaji}

Institute of Agriculture, The Graduate school of Tokyo, University of Agriculture and Technology, 3-5-8 Saiwai-cho, Fuchu,

Tokyo 183-8509, Japan
Table 2 Best Generalized Linear Mixed Models (GLMM) used to explain RAI of wild boars in Tochigi, central Japan.

\begin{tabular}{lllll}
\hline Variable & Estimate & SE & $\mathrm{z}$ & $P$-value \\
$($ Constant $)$ & -3.303 & 0.162 & -20.446 & $<0.001$ \\
Distance & 0.000 & 0.001 & 0.480 & 0.632 \\
Hunting season: Hunting & -1.174 & 0.165 & -7.112 & $<0.001$ \\
Region: Himuro & -0.042 & 0.263 & -0.158 & 0.875 \\
Time $:$ Night & 1.341 & 0.090 & 14.891 & $<0.001$ \\
Distance $\times$ Hunting & 0.001 & 0.000 & 2.314 & 0.021 \\
Distance $\times$ Region & -0.001 & 0.001 & -1.324 & 0.185 \\
Hunting $\times$ Region & -0.570 & 0.120 & -4.753 & $<0.001$ \\
Distance $\times$ Time & -0.005 & 0.001 & -8.820 & $<0.001$ \\
Hunting $\times$ Time & 0.779 & 0.168 & 4.645 & $<0.001$ \\
Region $\times$ Time & 0.183 & 0.139 & 1.316 & 0.188 \\
Distance $\times$ Region $\times$ Time & 0.003 & 0.001 & 3.905 & $<0.001$ \\
\hline
\end{tabular}

Italicized entries are variables with $P<0.05$ 